\title{
Implementation of Distributed Power Control /Active Link Protection for Femto Cell Networks
}

\author{
Sreevardhan Cheerla*, D. Venkata Ratnam, M. Siva Ganga Prasad, B. Vyshanvi and A. Sai Nitin \\ Department of ECE, KLEF, KL University, Vaddeswaram - 522502, Guntur, Andhra Pradesh, India; \\ sreevardhancheerla@gmail.com, ratnam2002v@gmail.com, alladafamily758@gmail.com
}

\begin{abstract}
Femto cell plays major role in the modern wireless communication systems applications. Femto cell provides higher data rates with a tiny base station. The prime concern in femto cell is power allocations for mobile receiver due to interferences. There is a need to develop a suitable power allocation algorithm to improve performance of femto cells. In this paper, an optimized power algorithm Distributive Power Control with Active Link Protection (DPC /ALP) is implemented. The proposed algorithm is tested under MATLAB simulated Long Term Evolution (LTE) environment and also evaluated with existing power allocation techniques i.e. fixed power scheme and power adaptive SINR. It is found that $39 \%$ improvement of power optimization is obtained when compared to the other techniques. The outcome of this research work would be immensely useful for developing robust power optimization techniques for femto cells
\end{abstract}

Keywords: Active Link Protection (ALP), Distributed Power Control, Femto Cells, LTE, SINR

\section{Introduction}

Recent studies show that over the last few decades there is tremendous increase in the mobile traffic rates in which $90 \%$ of the calls are from the indoor areas ${ }^{1}$. Femto cells are handy cells which help end users to upgrade the data transfer rates: as the received signal by the user is declined due to factors like interference, lower data rate transmission, path loss etc. These femto cells are particularly intended to use in the ranges of poor transmission and reception of the signals. Femto cells share its spectrum with the macro cell network which results in the cross-tier interference. Due to the random usage of these femto cells, the users might suffer from the interference from nearby femto cells. Interference is the key factor limiting the performance of the femto cells and results in the low Quality of Service (QOS) ${ }^{1}$. In order to avoid this interference problem we need to allocate power and bandwidth optimally to the femto cells. The Adaptive Distributed Femto cell Coverage Optimization
(ADFCO) is implemented for improvement in indoor coverage area. It is noticed that 50\% enhancement in power allocation and 1.6 times better indoor coverage area when compared to the fixed power transmission allocation scheme $e^{2}$. Adaptive uplink transmission scheme is implemented to avoid the interference between closed access femto cells to the other femto cells by allocating the orthogonal resources. Spectrum efficiency is increased by avoiding co-tier interference ${ }^{3}$. The Nash equilibrium power control reduced the power level but the satisfactory SIR is achieved ${ }^{4}$. The algorithm designed for the selfoptimization of Femto Base Station (FBS) yields better power optimization depending on the location of the cells ${ }^{5,6}$. DPC algorithm is developed, where the power is dynamically optimized ${ }^{7,8}$. In our work, the DPC algorithm is furthermore strengthened by introducing the DPC/ ALP (Active Link Protection). DPC/ALP algorithm is implemented to calculate the parameters which are required for computing the power updates. Such type of algorithms can be implemented independently without

\footnotetext{
${ }^{*}$ Author for correspondence
} 
depending upon the communication either with the base station or the user and such an algorithm is known as distributed.

\section{Power Control Algorithm}

Uplink power control or the downlink power control is considered for the analysis. The following are the parameters to be calculated in order to implement the 2.1DPC/ALP algorithm:

Path loss is the attenuation of electromagnetic wave when it is propagated through a medium (space). Simply, the variation between the transmitted power and received power is known as path loss?

Path loss $(\mathrm{PL})=10^{\star} \mathrm{n}^{\star} \log _{10} \mathrm{~d}[\mathrm{~m}]+\mathrm{L}_{\mathrm{f}}\left(\mathrm{n}_{\mathrm{f}}\right)$

$\mathrm{n}=$ Path loss exponent ( $\mathrm{n}=4$ in free space)

$\mathrm{d}=$ Distance in meters.

$\mathrm{L}_{\mathrm{f}}\left(\mathrm{n}_{\mathrm{f}}\right)=$ Attenuation.

Path loss between the user equipment and macro base station are as follows'

Path loss $($ Line of Sight $)=30.8+24.2 \log 10(\mathrm{R})+\mathrm{M}$

Path loss $($ Non- Line of Sight $)=2.7+42.8 \log 10(\mathrm{R})+\mathrm{M}$

$\mathrm{R}=$ Distance between User equipment and Base station.

$\mathrm{M}=$ Attenuation Parameter.

Similarly path loss for the femto base station and user equipment can be calculated as follows':

Path loss $=38.46+20 \log _{10}(\mathrm{R})+0.7 \mathrm{~d} 2 \mathrm{D}$, indoor $+18.3^{\star} \mathrm{n}$ $((\mathrm{n}+2) /(\mathrm{n}+1)-0.46)+\mathrm{q}^{*} \mathrm{~L}_{\text {iw }}$

Where,

$\mathrm{n}=$ No of floors or No of buildings.

$\mathrm{q}=$ Separation walls of $\mathrm{BS}$ and UE

$\mathrm{L}_{\mathrm{iw}}=$ Building wall penetration loss

The penetration loss that occurs due to the walls inside the apartment is given by the term $0.7 \mathrm{~d} 2 \mathrm{D}$, indoor. In case of single floor building we can ignore the last term $\mathrm{q}^{*} \mathrm{~L}_{\text {iw }}$.

SINR (Signal -to-Interference-Noise-Ratio) is used to measure the strength of the signal in a noisy environment at the receiver end. It gives the maximum capacity of channel. In general,

$\operatorname{SINR}=\frac{\mathrm{Rx}}{(\mathrm{I}+\mathrm{N})}$
Rx - Received Power

I - Interference of the signal

$\mathrm{N}$ - Noise generated.

In our algorithm we calculate the SINR as follows ${ }^{4}$ :

$\operatorname{SINR}=\frac{G(j) P(j)}{\sum_{j !=1}^{n} G(j) P(j)+N(j)}$

$\mathrm{n}=\{1,2,3 \ldots \mathrm{j}\}$

$\mathrm{G}=$ Channel Gain

Channel gain can be calculated according to the given equation

$G=10\left(-\frac{P L}{10}\right)$

Optimum power is achieved if the channel gain is estimated at each receiver for every received power.

Where $G(j)$ represents the channel gain of the $i^{\text {th }}$ user, $P(j)$ represents the power transmitted from the $j^{\text {th }}$ user and $\mathrm{N}(\mathrm{j})$ represents the noise at the $\mathrm{i}^{\text {th }}$ user.

\section{DPC/ALP Algorithm}

Step 1: Initialize the parameters.

(Minimum transmission power, Target SINR, Maximum transmission power)

Step 2: Calculation of Path loss according to (4).

Step 3: Calculation of SINR according to (6).

Step 4: For $\mathrm{i}=1$ : number of femto cells (updating the powers)

If Current SINR > Target SINR

Power $=\min \left((1+€)^{\star}(\text { Target SINR/Current SINR })^{\star}\right.$ last femto power, max power)

Else

Power $=(1+€)^{*}$ last femto power

Where $€$ is SINR margin

SINR margin helps to protect the active users from the new users.

\section{Results and Discussion}

The Femto Base Station (FBS) transmitted power is calculated by using four different power schemes, such as: Fixed Power Transmission where each femto base station is assigned with the same power, Power Adaptive Range which mainly focus on achieving the constant femto cell 
radius, Power adaptive SINR where the power is allocated to each base station based upon the SINR values and DPC/ ALP scheme is an iterative algorithm, which changes the power values dynamically depending on the condition $(8)^{11}$. Simulations are performed for every type of power scheme and presented.

The software used for simulating the power schemes is "Femto cell Power Management Interference Simulator $(\text { FePIS })^{12}$. In this simulation we compare different power schemes models with respect to basic network parameters such as path loss, throughput, SINR, power. Simulations are performed, each time ensuring different power scheme.

\subsection{Simulation Considerations}

Macro BS Allocated: 1

Femto BS Allocated: 4

Macro users Allocated: 4

Femto users Allocated: 4

Bandwidth/Modulation Scheme: 20MHz/64QAM

The parameters considered for simulation in LTE simulator are listed in Table 1.

Table 1. LTE Simulation parameters

\begin{tabular}{llc}
\hline Sl.No & Parameter & Value \\
\hline 1 & Cell layout & Hexagonal grid \\
2 & Macro Base Station Power & $46 \mathrm{dBm}$ \\
3 & Range of femto & $2 \mathrm{~m}$ \\
4 & Frequency & $2 \mathrm{GHz}$ \\
5 & Femto Base Station max power & $21 \mathrm{dBm}$ \\
6 & Femto Base Station default power & $11 \mathrm{dBm}$ \\
7 & Noise Power Density & $174 \mathrm{dBm} / \mathrm{Hz}$ \\
8 & Target SINR Range & $10 \mathrm{~m}$ \\
9 & Target SINR & $4 \mathrm{~dB}$ \\
10 & Outdoor wall loss & $15 \mathrm{~dB}$ \\
11 & Indoor wall loss & $7 \mathrm{~dB}$ \\
12 & FFR/ SFR Radius & $125 \mathrm{~m}$ \\
13 & Road width & $5.0 \mathrm{~m}$ \\
14 & Bandwidth & $20 \mathrm{MHz}$ \\
15 & Subcarrier Spacing & $15 \mathrm{KHz}$ \\
\hline
\end{tabular}

Figure 1 displays the basic layout of LTE-Simulator. It is Graphical User Interface (GUI) where we can create virtual network by using some parameters such as number of femto cells, number of femto users, numbers of macro cells, penetration floors etc. LTE simulator produces the SINR, path loss and throughput over various femto cell coverage areas in terms of local coordinates $(\mathrm{x}, \mathrm{y})$. To represent the attenuation factors Num. of buildings ( $\mathrm{x}$ ), Num. of buildings ( $y$ ) are included in GUI.

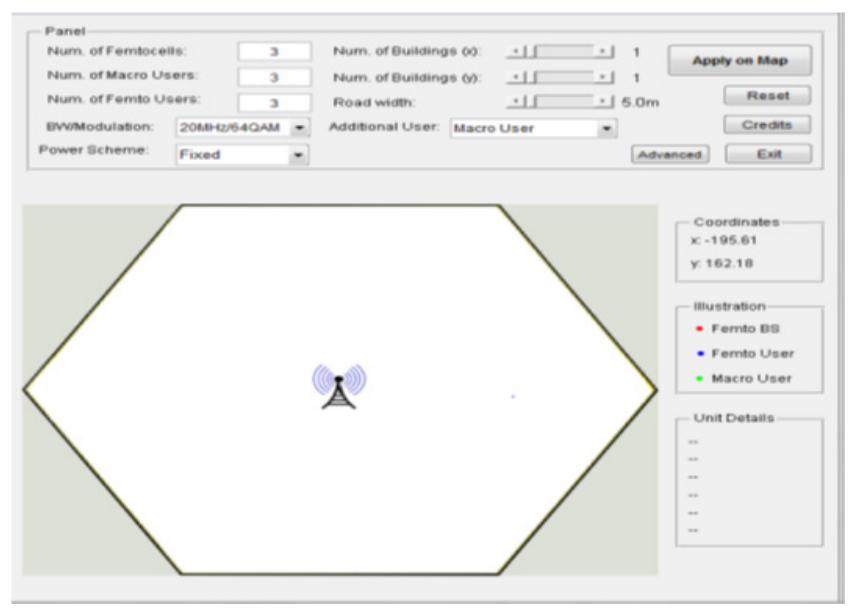

Figure 1. Basic Layout of LTE Simulator.

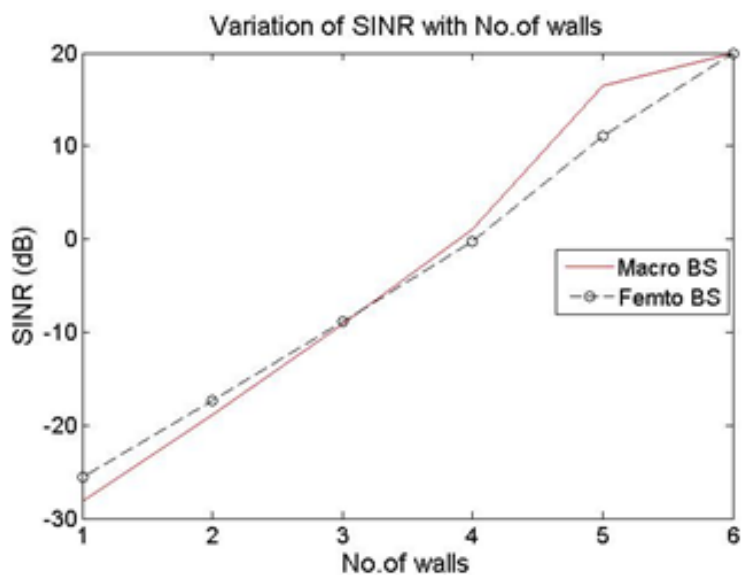

Figure 2. Variation of SINR with Number of walls.

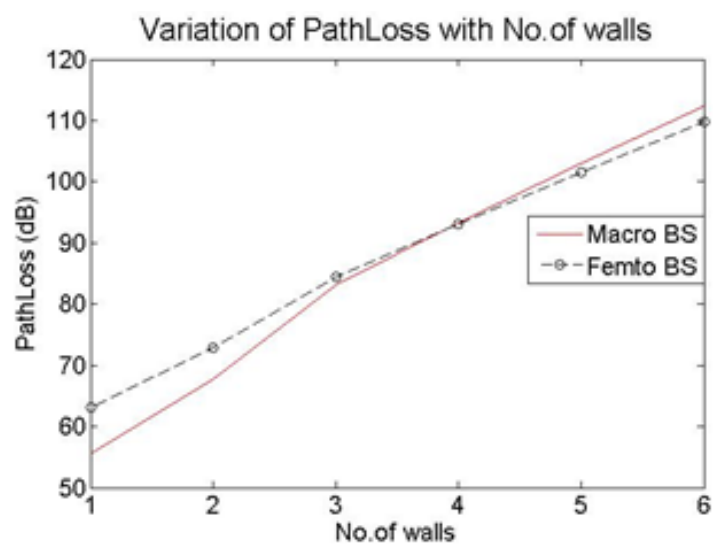

Figure 3. Variation of Path Loss with Number of walls. 


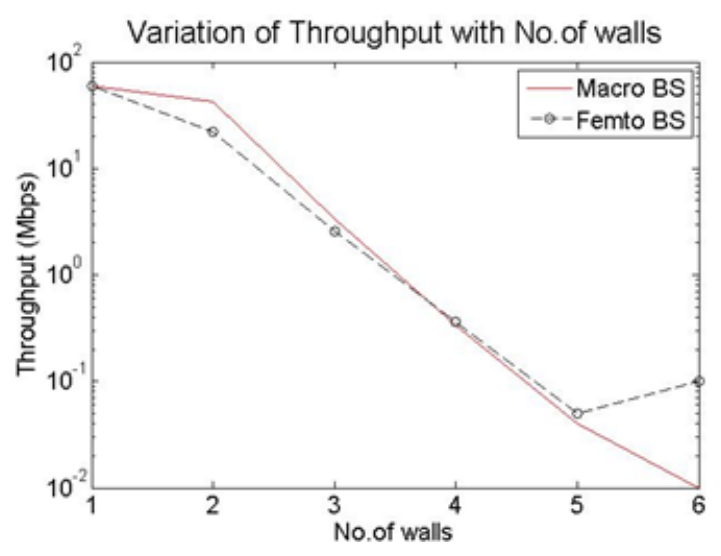

Figure 4. Variation of Throughput with Number of walls.

Figure 2 shows SINR results of LTE simulator. As the number of walls increases the SINR also increases. From Figure 2 it is clear that, up to 3 walls the signal strength of Femto Base station radio link is stronger. As the radio link crosses more than 3 walls then Macro Base station radio link becomes stronger ${ }^{10}$. Figure 3 Displays the variation of Path loss with No. of walls. It is similar to the above case (Figure 2). Up to 3 walls Femto Base Station radio signal is stronger; when the signal crosses more than 3 walls then the Macro Base Station radio signal becomes stronger. Figure 4 displays the variation of throughput with number of walls. The variation of throughput and SINR are contrarily corresponding in such a way that up to 3 walls between the user equipment and base station, Macro Base Station gives the better Radio link. Once the number of walls exceeds 3, the Femto Base Station turns out to be having better throughput ${ }^{10}$. This gives a trade off in the middle of throughput and SINR for Femto Base Station and Macro Base Station.

The network parameters for femto and Macro base stations are listed in Table 2.

Table 2. Network Parameters for Femto and Macro Base Stations

\begin{tabular}{lccc}
\hline $\begin{array}{l}\text { LTE-A Network } \\
\text { Prameters }\end{array}$ & $\begin{array}{c}\text { No. of } \\
\text { walls }\end{array}$ & $\begin{array}{c}\text { Femto BS } \\
\text { Link }\end{array}$ & $\begin{array}{c}\text { Macro BS } \\
\text { Link }\end{array}$ \\
\hline \multirow{2}{*}{ SINR } & $<=3$ & High & Low \\
& $>4$ & Low & High \\
Throughput & $<=3$ & Low & High \\
& $>4$ & High & Low \\
Path Loss & $<=3$ & High & Low \\
& $>4$ & Low & High \\
\hline
\end{tabular}

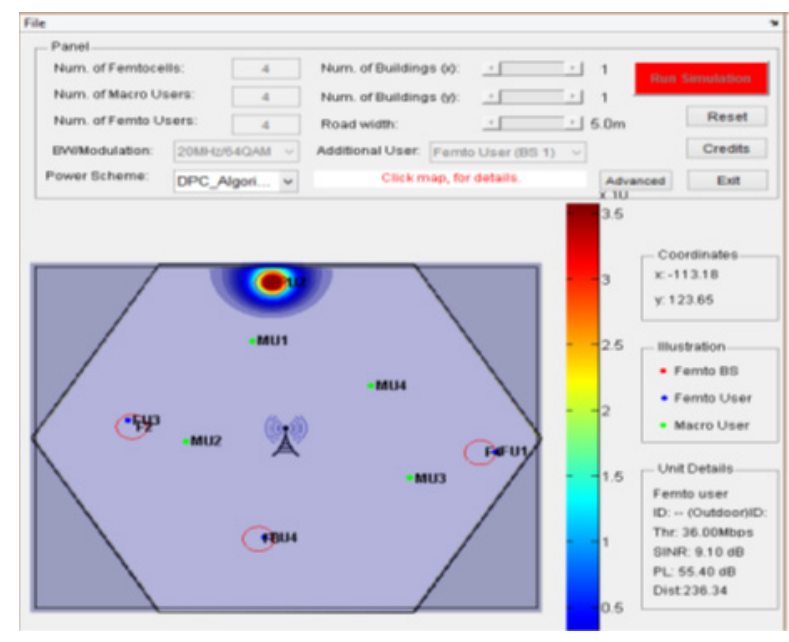

Figure 5. LTE-Simulator Graphical User Interface with DPC/ALP Scheme.

Figure 5 Displays the DPC/ALP scheme. It is an iterative algorithm where each base station updates its power values according to the SINR values. In this power scheme SINR margin helps to protect active users from the new users.

The signal strength variations are shown in Figure 5 for an additional user (femto user BS1). In the figure the amount of signal reception surrounding the femto cell region varies with the color as Red $>$ Yellow $>$ Cyan $>$ Blue. The intensity is varied from 0.5 to $3.5 \mathrm{~dB}$.

The throughput, SINR and the path loss for the femto user BS1 are 36.00 Mbps, $9.10 \mathrm{~dB}$ and $55.40 \mathrm{~dB}$ respectively. Similarly, all these parameters can be computed for each and every user BS in the LTE simulator.

The power values achieved through the DPC / ALP are listed in Table 3.

Table 3. DPC/ALP input/output power values

\begin{tabular}{lllll}
\hline Power Allocated to each & 0.0631 & 0.0631 & 0.0631 & 0.0631 \\
FBS & & & & \\
Power used by each FBS & 0.0155 & 0.0406 & 0.0818 & 0.0162 \\
\hline
\end{tabular}

Power Comparison of different Power schemes is listed in Table 4.

Figure 6 depicts the plot corresponding to the methodologies mentioned in our paper i.e. Fixed Power Scheme, Power Adaptive SINR, Power Adaptive Range, DPC/ALP schemes. From the figure it is evident that, the DPC/ALP scheme utilizes low power. 
Table 4. Different Power Schemes simulated values

\begin{tabular}{llcccc}
\hline Sl.No & \multicolumn{1}{c}{ Power Scheme } & $\begin{array}{c}\text { Power of FBS1 } \\
\text { (in dB) }\end{array}$ & $\begin{array}{c}\text { Power of FBS2 } \\
\text { (in dB) }\end{array}$ & $\begin{array}{c}\text { Power of FBS3 } \\
\text { (in dB) }\end{array}$ & $\begin{array}{c}\text { Power of FBS4 } \\
\text { (in dB) }\end{array}$ \\
\hline 1 & Fixed Power & 0.0631 & 0.0631 & 0.0631 & 0.0631 \\
2 & Power Adaptive Range & 0.0484 & 0.0803 & 0.1259 & 0.0339 \\
3 & Power Adaptive SINR & 0.0946 & 0.1420 & 0.2129 & 0.0339 \\
4 & DPC/ALP Scheme & 0.0155 & 0.0406 & 0.0818 & 0.0162 \\
\hline
\end{tabular}

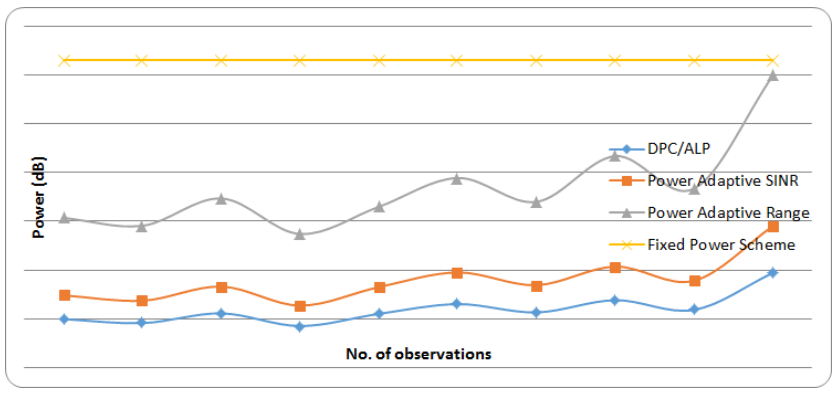

Figure 6. LTE-Simulator Graphical User Interface with DPC/ ALP Scheme.

\section{Conclusion and Future Work}

Femto cells play a vital role in strengthening the transmitted signal with less power usage by the femto cell. Though the coverage area by a femto cell is increased, the hindrance is the interference level; this can be overcome with help of dynamically changing DPC/ALP scheme. Out of all the considered power schemes, power utilized by the DPC/ALP scheme is low, this in turn can optimize the interference levels. The amount of enhancement by DPC/ALP for first femto BS is $83 \%$ more than the Power Adaptive SINR at a distance of $236.34 \mathrm{~m}$ from the BS. Similarly, the percentage of enhancement varies with the distance of the user from the femtoBS. This analysis can be extended for femto cell coverage analysis.

\section{Acknowledgement}

The work of D. V. Ratnam was supported through F. 301/2013(SAII)/RA201416GEANP5585.

\section{References}

1. Zheng W, et al. Distributed power optimization for spectrum-sharing femtocell networks: A fictitious game ap- proach. Journal of Network and Computer Applications. 2014; 37:315-22.

2. Valizadeh SR, Abouei J. An adaptive distributed coverage optimization scheme in LTE enterprise femtocells. 2014 22nd Iranian Conference on Electrical Engineering (ICEE); IEEE; Iran. 2014. p. 1723-8.

3. Saleem S, King H. Avoidance of co-tier interference between femtocells with different access modes. International Journal of Informationand Communication Research. 2012; 2(8):617-26.

4. Nazmul H, Rahim A, Azam S. SIR-Based Power Control Algorithms in CDMA Networks. Global Journal of Computer Science and Technology. 2013; 13(10):40-5.

5. Tsao K-J, Shen S-C, Hou TC. Location-dependent power setting for next generation femtocell base stations.2011 IEEE Wireless Communications and Networking Conference (WCNC); Cancun: Quintana Roo. 2011. p. 767-72.

6. Saad SA, Ismail M, Nordin R. A survey on power control techniques in femtocell networks. Journal of Communications. 2013; 8(12):845-854.

7. Agarwal A, Dahal P, Poonam S. Adaptive Power Control Applying to Femto cell. National Institute of Technology. Rourkela. 2013.

8. Jeffrey M. Distributed Power Control Algorithms in Wireless Networking. 2009.

9. Mung C, et al. Power control in wireless cellular networks. Foundations and Trends ${ }^{\circledast}$ in Networking. 2008; 2(4):381533.

10. Vardhan CS, et al. Analysis of path loss models of $4 \mathrm{G}$ fem to cells. 2014 Eleventh International Conference on Wireless and Optical Communications Networks(WOCN); India. 2014. p. 1-6.

11. Bouras C, et al. Power management over co-channel femtocells in LTE-A systems. 2012IFIP WirelessDays(WD)IEEE; 2012.p. 1-3.

12. Femtocell Power Management Interference Simulator (FePIS). Available from: http://ru6.cti.gr/ru6.20.04.2015 